\title{
APPLICATION OF MACHINE LEARNING IN URBAN GREENERY LAND COVER EXTRACTION
}

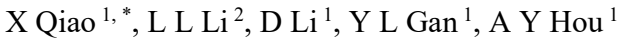 \\ ${ }^{1}$ Qingdao Geotechnical Investigation and Surveying Institute, State and Local Joint Engineering Research Center for the Integration \\ and Application of Sea-land Geographical Information, Shandong Road, Qingdao, China - (49984066@qq.com, \\ 155171408@qq.com, qdprs201601@163.com, houanye@126.com) \\ ${ }^{2}$ College of Information Science and Engineering, Ocean University of China, Songling Road, Qingdao, China - lilele@ouc.edu.cn
}

\section{Commission III, WG III/1}

KEY WORDS: Neural Network, Machine Learning, Greenery Land Cover, Auto Extraction, Multispectral Image

\begin{abstract}
:
Urban greenery is a critical part of the modern city and the greenery coverage information is essential for land resource management, environmental monitoring and urban planning. It is a challenging work to extract the urban greenery information from remote sensing image as the trees and grassland are mixed with city built-ups. In this paper, we propose a new automatic pixel-based greenery extraction method using multispectral remote sensing images. The method includes three main steps. First, a small part of the images is manually interpreted to provide prior knowledge. Secondly, a five-layer neural network is trained and optimised with the manual extraction results, which are divided to serve as training samples, verification samples and testing samples. Lastly, the well-trained neural network will be applied to the unlabelled data to perform the greenery extraction. The GF-2 and GJ-1 high resolution multispectral remote sensing images were used to extract greenery coverage information in the built-up areas of city X. It shows a favourable performance in the 619 square kilometers areas. Also, when comparing with the traditional NDVI method, the proposed method gives a more accurate delineation of the greenery region. Due to the advantage of low computational load and high accuracy, it has a great potential for large area greenery auto extraction, which saves a lot of manpower and resources.
\end{abstract}

\section{INTRODUCTION}

Urban greenery (i.e., street trees, shrubland, grassland, and other forms of vegetation) has long been recognized as a critical part of the modern city (Wong and Chen, 2010). The greenery coverage information plays a vital role in land resource management, environmental monitoring and urban planning. For example, the greenery coverage area is necessary to calculate the greenery coverage ratio which is an important index in evaluating national ecological garden city.

But due to the wide and complex distribution of greenery in city, the automatic extraction methods to produce such data has been an intense reserach focused in recent decades. The most popular way of vegetation extraction is by using Normalized Difference Vegetation Index (NDVI) (Rouse Jr et al., 1974). NDVI is calculated from a normalized transform of the near-infrared (NIR) and red reflectance ratio, which reflects the absorptive and reflective characteristics of vegetation in those two spectra. Based on a similar principle, many other vegetation indexes are proposed for vegetation extraction, such as SAVI (Huete, 1988), NDWI (Gao, 1996), CI (Vescovo and Gianelle, 2008).

However, the NDVI and other mentioned vegetation indexes are, to some extent, too simple to present complex situation in real applications. The vegetation index based method turns out to be sensitive to atmospheric effect, clouds, soil effect and anisotropic effect. Thus, poor classification accuracy is usually resulted, particularly in the inhomogeneous region such as urban area. Also, a different threshold is needed for different situations, which requires plenty of professional knowledge.

To fill the above mentioned gaps in the vegetation extraction method using vegetation index, we introduced a new automatic method based on neural network for urban greenery extraction from multispectral satellite images. The idea of neural network was developed by Rosenblatt (1958) who was inspired by McCulloch et al.'s (1943) earlier work. It is a basic machine learning method which can solve the supervised classification method by learning from training samples. It has been increasingly applied to remote sensing imagery classification (Giacinto and Roli, 2001; Heermann and Khazenie, 1992; Tzeng and Chen, 1998). In this research, the neural network is extended to extract greenery in different scenarios including the difficult cases where greenery is dispersive and highly mixed with the built-ups in the modern city.

The following part of this paper is organized as follows. A description of the multispectral satellite data used in this research is provided in Section 2. Section 3 presents the details of the proposed neural network method for urban greenery extraction. Section 4 shows the extraction results conducted on GF-2 and GJ-1 multispectral data, as well as the comparison between our proposed method and traditional NDVI method. The summary and conclusions are presented in the last section.

\section{DATASET DESCRIPTION}

\subsection{Working Area}

The working area covers more than 600 square kilometers, and the greenery regions mainly include the cropland, forest, grassland and shrubland. The none-greenery regions, which are basically the built-ups, include buildings, roads, water and other communal facilities. Gaofen-2 (GF-2) and Gaojing-1 (GJ-1) multispectral images were used in this research. The multispectral images contains R, G, B and NI bands, but for 
easy display, only the visible light image with $\mathrm{R}, \mathrm{G}$ and $\mathrm{B}$ bands of the data are shown in this paper.

\subsection{GaoFen-2 Data}

GaoFen-2 is a follow-on mission of the Gaofen-1 technology demonstration mission. It was launched in 2014 on a CZ-4B rocket from China's Taiyuan space centre. It is an optical satellite with a high resolution imaging payload, which is capable of collecting images with a resolution of $0.81 \mathrm{~m}$ in panchromatic mode and $3.24 \mathrm{~m}$ in the multispectral mode. 3 GF-2 images acquired from March to May 2017 were used in this research as Figure 1 shows.

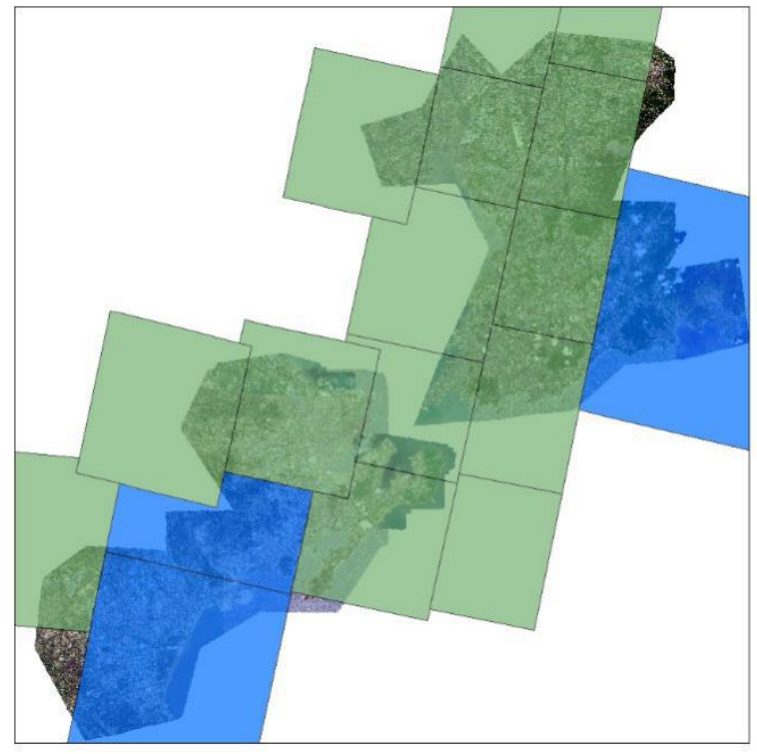

Figure 1. Satellite images used in this work

\subsection{GaoJing-1 Data}

GaoJing-1, which is also called SuperView-1, is a commercial constellation of Chinese remote sensing satellites operated by Beijing Space View Technology Co. Ltd. It was launched on December 28, 2016. This satellite is to provide imagery with 0.5 $\mathrm{m}$ panchromatic resolution and $2 \mathrm{~m}$ multispectral resolution. 14 SuperView images acquired from May to June 2017 were used in this research as Figure 1 shows.

\section{MEHTODOLOGY}

\subsection{Overview}

The automatic greenery extraction method is developed based on neural network. It follows the basic supervised machine learning process. The method includes three main steps. First, a small part of the image is manually interpreted to provide prior knowledge. Secondly, a five-layer neural network is trained and optimised with the manual extraction results. Lastly, the well-trained neural network is applied to the unlabelled data to perform the pixel-level greenery extraction.

\subsection{Training Sample Preparation}

A small part of the multispectral data with the corresponding manually interpreted extraction results is needed to train the neural network. Figure 2 illustrates the training sample preparation. Figure 2 (a) shows the unlabelled remote sensing data. A small part of the data (as Figure 2 (b) shows) was selected for the training purpose. Figure 2 (c) shows the manually marked ground truth, with 0 (marked as black) indicating the greenery areas and 1 (marked as white) indicating the none-greenery areas. In the following training process, $70 \%$ of the training datasets were used for training, $15 \%$ for validation and $15 \%$ for testing. In this working area, an area of $300 \times 500$ pixels was selected for training purpose. The size is the optimal choice which is enough to acquire a well-trained neural network model and to cost less human works in training sample preparation. If a larger-size training data was prepared, slightly better performance would be expected.

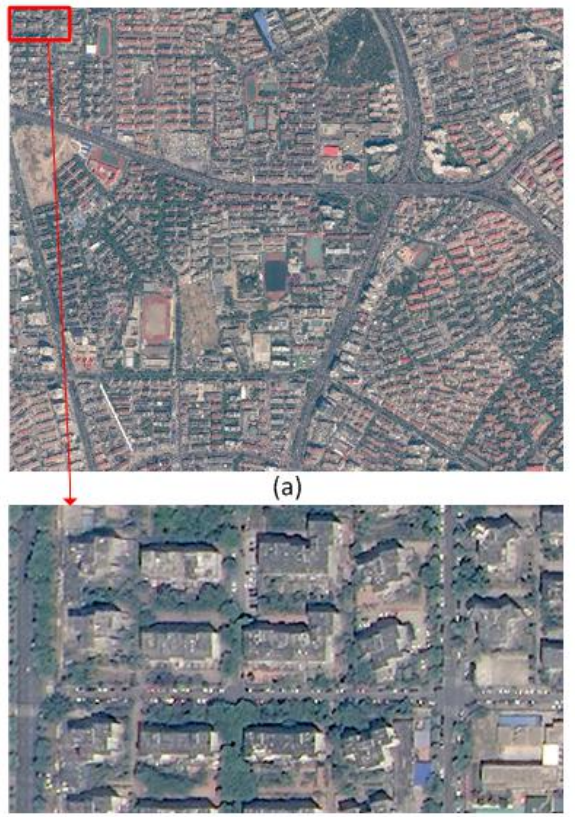

(b)

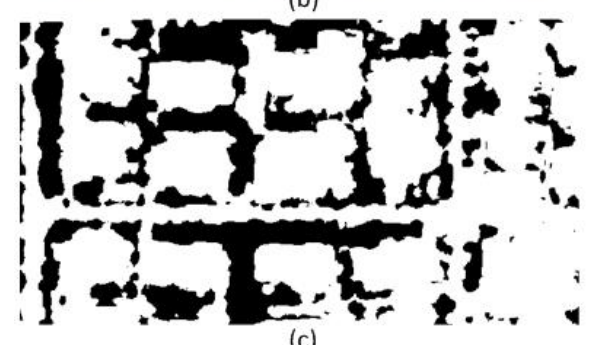

Figure 2. Illustration of the training sample

\subsection{Neural Network}

A fully interconnected back propagation neural network with 5 layers were used to solve this pixel level binary classification problem. The model was developed based on Neural Network Toolbox in Matlab R2017b. Multispectral imagery consisting of the visible spectral bands red, green and blue, and the near-IR band 4 was used in the work.

The structure of the model is illustrated in Figure 3. The first layer is the input layer, composes of a $m$ by $n$ by 4 arrays of neurons used for processing input. The $m$ and $n$ are the width and height of the input image. The following three layers are processing layers or so-called "hidden" layers. Several researchers have found that the number of hidden layers greatly affect the performance of neural networks. According to experimental experience, three-hidden-layers is the optimal choice in this work. Fewer layers is not sufficient to model the complex situation. Meanwhile, too many layers will lead to overfitting problem and also increase processing time. The output layer was composed of two neurons representing the two 
classes, which indicate greenery and none-greenery area. Every neuron within one layer is fully interconnected with those neurons in the adjacent layers. These interconnections are adaptive coefficients between the node $i$ in current layer and node $j$ in the previous layer in the network. The collection function (Rumelhart et al., 1987) between node $i$ and node $j$ is as follows:

$$
\text { net }_{i}=\sum_{j=1}^{n} W_{j i} O_{j}+\text { bias }_{i}
$$

where

$$
\text { net }_{i}=\text { the result of the collection function }
$$

$W_{j i}$, bias $_{i}=$ the weight and bias of the interconnection from current node $i$ to the node $j$ in the previous layer state.

$$
O_{j}=\text { the sigmoid function to determine the output }
$$

All the nodes in the current layer will be connected to the node in the previous layer according to this collection function. The weights and biases will be adjusted iteratively with the training data to improve the overall performance of the model.

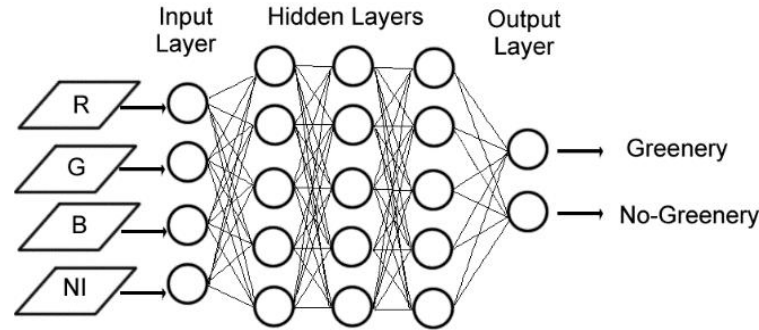

Figure 3. The structure of the proposed neural network model

In our algorithm, the data used for training, testing and verification is divided by random. Levenberg-Marquardt is used as the cost function and the performance of the model is evaluated by mean squared error (MSE). Figure 4 shows the plot of MSE in each epoch when training the pilot area of this work. As we can see, the model converges quickly within only 15 iterations. It reaches the best performance of 0.0724 in 34 epochs and shows no improvement afterward.

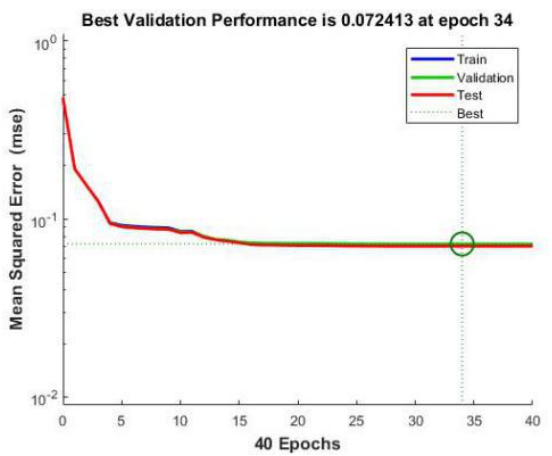

Figure 4. The plot of MSE in each epoch when training the pilot area

\subsection{Extraction}

Once the neural network is well trained, it can be applied to other multispectral remote sensing data. We use 0 and 1 to indicate greenery and none-greenery area in training process, thus the output is two groups of numbers around 0 and 1 respectively. 0.5 is set as a threshold to split the output into two categories. The model only needs to be retrained when there is significant changes in weather condition and atmosphere, or when another different source data is used.

\section{RESULTS}

\subsection{Experimental Results}

The proposed method was applied and tested on two sets of data, which are GF-2 and GJ-1 multispectral satellite image as illustrated in the previous section. Figure 5 and Figure 6 show the extraction results of GF-2 data and GJ-1 data. In both Figure 5 and Figure 6, (a) is part of the original dataset (optical bands). Two specific areas are enlarged and displayed in (c) and (e). The corresponding detection results are shown in (b), (d) and (f), with the blue colour indicates the greenery extraction results. It can be seen that the proposed method successfully extract the greenery from the background. The edge of the greenery is also well delineated.
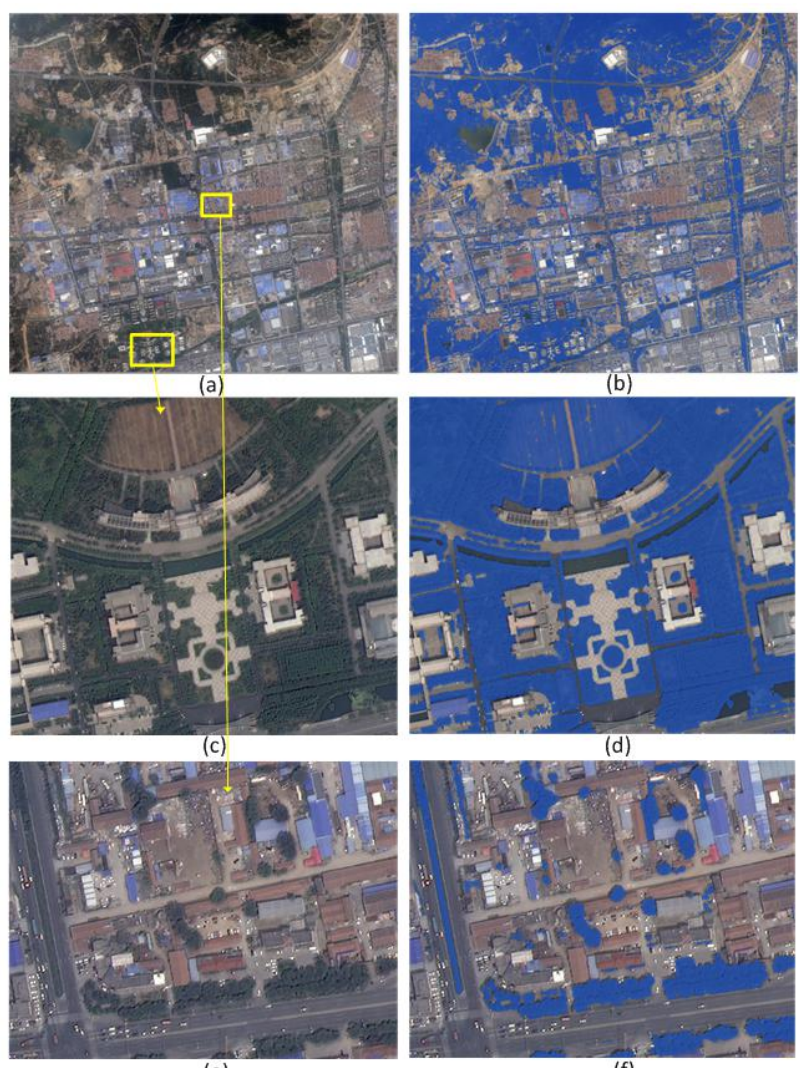

(e)

(f)

Figure 5. Greenery extraction result of GF-2 image 

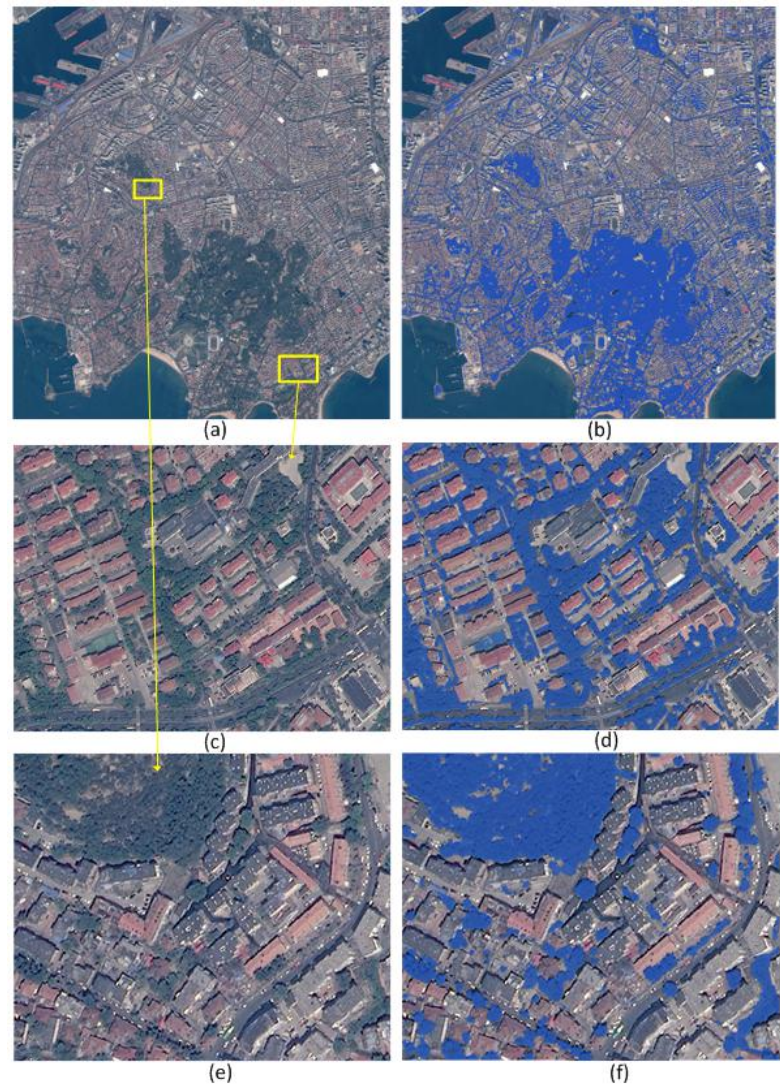

Figure 6. Greenery extraction result of GJ-1 image

\subsection{Comparison with NDVI Method}

To further evaluate the performance of the proposed greenery extraction method, the extraction results were compared with the traditional NDVI method. The NDVI is calculated as follows:

$$
N D V I=\frac{(N I R-R E D)}{(N I R+R E D)}
$$

where $\quad N I R=$ the value of near-infrared band $R E D=$ the value of red band.

Calculations of NDVI for a given pixel will result in a number that ranges from -1 to +1 . Zero means none-vegetation and close to $+1(0.8-0.9)$ indicates the highest possibility of a vegetation area. In the experiment, the threshold for vegetation and none-vegetation is selected according to experimental experience.

In Figure 7, (a) is the original image displays as reference, (b) shows the greenery extraction result using NDVI, (c) shows the result of the proposed neural network based method. It is apparent from Figure 7 that the shape of the vegetation is better extracted by the proposed method than the traditional NDVI method. As marked by yellow rectangles, many street pixels are wrongly marked as greenery by NDVI method. In addition, as highlighted by red rectangles, our proposed method successfully extracted the greenery where NDVI method failed to do so.

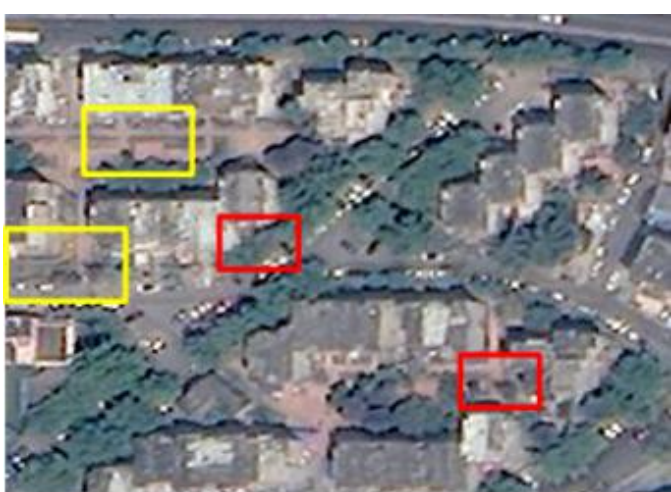

(a)

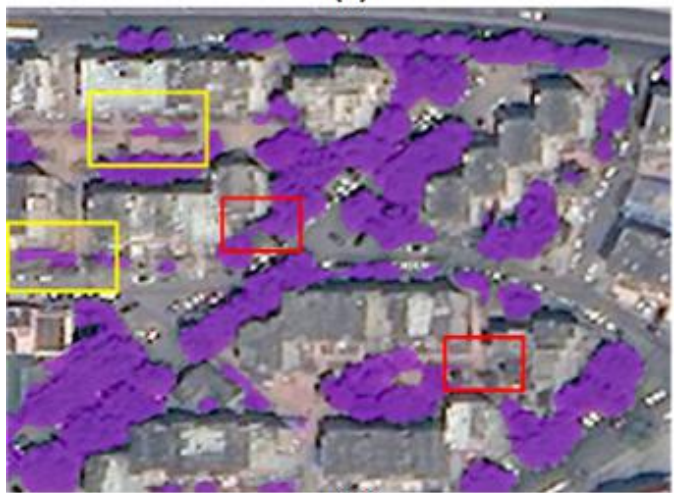

(b)

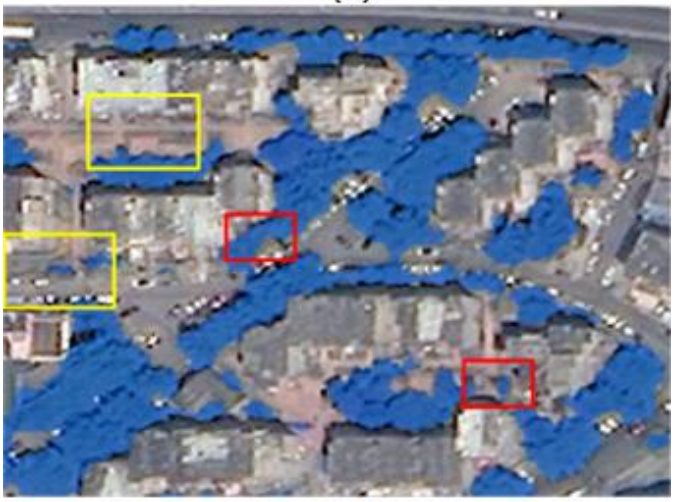

(c)

Figure 7. Comparison of greenery extraction results between the proposed method and NDVI method

\subsection{Application Results}

After precision and efficiency validation of the proposed method, we applied the neural network model in the greenery extraction in built-up areas of city X. The area covers 619 square kilometers, and the results are shown in Figure 8.. 


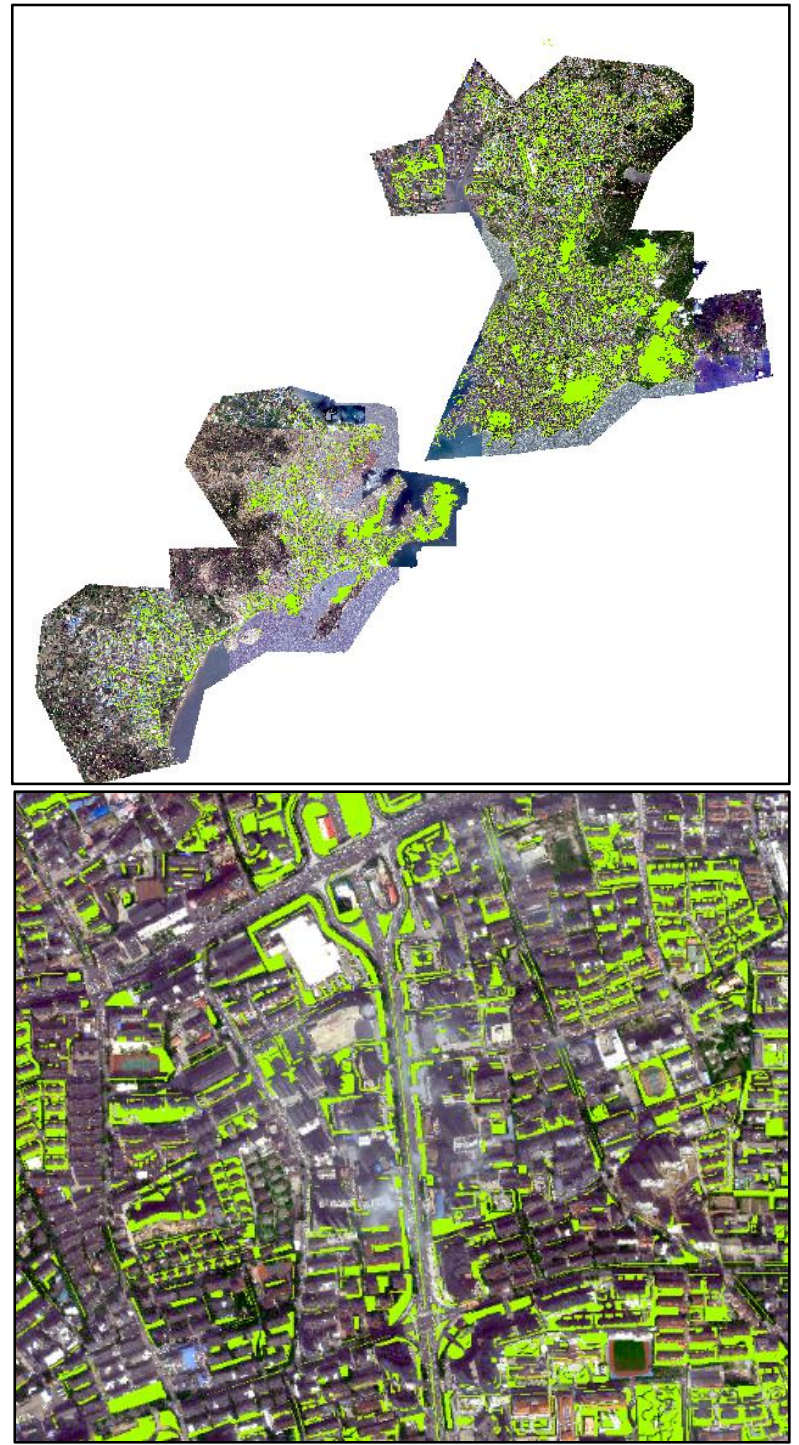

Figure 8. Greenery extraction result in built-up areas of city X

The whole process including training sample preparation and automatic extraction cost 10 days while it would cost 170 days to manually interpret the greenery area.

\section{CONCLUTIONS AND FUTURE WORK}

In this work, we proposed a new method for automatic urban greenery extraction from multispectral satellite image. Considering the complex situations in the city, instead of using the simple vegetation index, the supervised machine learning method neural network is extended to conduct the greenery extraction. We carried out a number of experiments to assess the performance of the proposed approach, also in comparison with the traditional NDVI based method. Experimental results show that the proposed method can extract greenery in different scenarios including the difficult case where greenery is dispersive and highly mixed with the built-ups in the downtown area. And our extraction results in city X built-up areas over 600 square kilometers showed a better performance than traditional ways.

For future work, the performance of the neural network model can be enhanced by including more representative areas into the training datasets. In addition, the method also has a potential for the extraction of other objects, such as water and road.

\section{REFERENCES}

Gao, B.-C., 1996. NDWI-A normalized difference water index for remote sensing of vegetation liquid water from space. Remote sensing of environment 58, 257-266.

Giacinto, G., Roli, F., 2001. Design of effective neural network ensembles for image classification purposes. Image and Vision Computing 19, 699-707.

Heermann, P.D., Khazenie, N., 1992. Classification of multispectral remote sensing data using a back-propagation neural network. IEEE Transactions on Geoscience and Remote Sensing 30, 81-88.

Huete, A.R., 1988. A soil-adjusted vegetation index (SAVI). Remote sensing of environment 25, 295-309.

McCulloch, W.S., Pitts, W., 1943. A logical calculus of the ideas immanent in nervous activity. The bulletin of mathematical biophysics 5, 115-133.

Rosenblatt, F., 1958. The perceptron: a probabilistic model for information storage and organization in the brain. Psychological review $65,386$.

Rouse Jr, J.W., Haas, R., Schell, J., Deering, D., 1974. Monitoring vegetation systems in the Great Plains with ERTS.

Rumelhart, D.E., McClelland, J.L., Group, P.R., 1987. Parallel distributed processing. MIT press Cambridge, MA.

Tzeng, Y.-C., Chen, K.-S., 1998. A fuzzy neural network to SAR image classification. IEEE Transactions on Geoscience and remote Sensing 36, 301-307.

Vescovo, L., Gianelle, D., 2008. Using the MIR bands in vegetation indices for the estimation of grassland biophysical parameters from satellite remote sensing in the Alps region of Trentino (Italy). Advances in Space Research 41, 1764-1772.

Wong, N.-H., Chen, Y., 2010. The role of urban greenery in high-density cities. Designing high-density cities for social and environmental sustainability, 227-262.

Revised March 2018 